\title{
Surface contribution to electronic density of states, heat capacity, and spin susceptibility
}

\author{
V. E. Kenner* and R. E. Allen \\ Department of Physics, Texas A\&M University, College Station, Texas 77843 \\ (Received 22 April 1974)
}

\begin{abstract}
The surface contributions to the electronic heat capacity $C_{\mathrm{el}}^{s}$ and spin susceptibility $\chi^{s}$ are, respectively, $C_{\mathrm{el}}^{s}=(1 / 3) A\left[\gamma\left(k_{F}\right)-\pi / 4\right] k_{B}^{2} T$ and $\chi^{s}=\left(\mu_{B}^{2} / \pi^{2}\right) A\left[\gamma\left(k_{F}\right)-\pi / 4\right]$ for any semi-infinite free-electron model, where $\gamma\left(k_{F}\right)$ is a phase shift determined by the surface potential. The electronic surface heat capacity thus can provide some information about the surface potential seen by electrons (or with many-body effects included, electron quasiparticles).
\end{abstract}

Recently Denton, Mühlschlegel, and Scalapino ${ }^{1}$ considered the heat capacity and spin susceptibility of small metallic particles, taking into account the fact that the electronic energy levels are discrete. The average spacing between levels at the Fermi surface, $\delta$, is given by $1 / \rho\left(E_{F}\right)$, where $\rho(E)$ is the density of states and $E_{F}$ is the Fermi energy。 For particles which are about $100 \AA$ in each dimension, $\delta / k_{B} \sim 1{ }^{\circ} \mathrm{K}$, where $k_{B}$ is the Boltzmann constant. Their results indicate that if $k_{B} T \leqslant \frac{1}{2} \delta$, where $T$ is the temperature, the discreteness of the spectrum has a significant effect on the physical quantities.

However, if $k_{B} T>\delta$, it should not be a bad approximation to neglect the discreteness of the spectrum. We therefore consider the surface contributions to the heat capacity and spin susceptibility for a semi-infinite crystal, with the attitude that our results can be applied to small particles if $k_{B} T>\delta$; i. e.,$T>1^{\circ} \mathrm{K}$ for $100-\AA$ particles. For metal foils which have a small thickness but are large in the other dimensions, the level spacing will be very small and our results will be valid even at very low temperatures.

Lang has obtained an expression for the number of electrons in a metal ${ }^{2}$ which we easily generalize to obtain

$$
N(k)=\frac{V}{3 \pi^{2}} k^{3}+\frac{A}{\pi^{2}} \int_{0}^{k} k_{z}\left(\gamma\left(k_{z}\right)-\frac{\pi}{4}\right) d k_{z},
$$

where $N(k)$ is the number of electronic states lying inside a sphere of radius $k$. Here $V$ is the volume, $A$ is the surface area, and $\gamma\left(k_{z}\right)$ is a phase shift defined by

$$
\psi_{k_{x} k_{y} k_{z}}(\overrightarrow{\mathrm{r}})-\sin \left[k_{z} z-\gamma\left(k_{z}\right)\right] e^{i\left(k_{x} x+k_{y} y\right)}
$$

as $z--\infty-i$. e. , far back inside the metal away from the surface. The potential seen by an electron near the surface determines $\gamma\left(k_{z}\right)$. Lang and $\mathrm{Kohn}^{3}$ have calculated the electronic wave functions $\psi$, and thus this phase shift, self-consistently within the framework of certain approximations, e.g. , the use of a local potential for exchange and correlation. This phase shift can also be determined for other model-surface barriers, such as an in- finite barrier or a finite-step barrier. The results (1) and (2) are valid for any semi-infinite free-electron model, ${ }^{4}$ i. e., any model in which the potential depends only on $z$ and approaches a constant value as $z \rightarrow-\infty$. Also for this model,

$$
E(\overrightarrow{\mathrm{k}})=\hbar^{2} k^{2} / 2 m \text {. }
$$

We have

$$
\begin{aligned}
\rho(k) & \equiv \frac{d N}{d k} \\
& =\frac{V}{\pi^{2}} k^{2}+\frac{A}{\pi^{2}} k\left(\gamma(k)-\frac{\pi}{4}\right),
\end{aligned}
$$

so that the density of states function is

$$
\begin{aligned}
\rho(E) & =\rho(k) \frac{d k}{d E} \\
& =\frac{m}{\hbar^{2}} \frac{V}{\pi^{2}} k+\frac{m}{\hbar^{2}} \frac{A}{\pi^{2}}\left(\gamma(k)-\frac{\pi}{4}\right) .
\end{aligned}
$$

The electronic heat capacity $C_{\mathrm{e} 1}$ and spin susceptibility $\chi$ are given by ${ }^{5}$

$$
\begin{aligned}
& C_{e 1}=\frac{1}{3} \pi^{2} \rho\left(E_{F}\right) k_{B}^{2} T, \\
& \chi=\mu_{B}^{2} \rho\left(E_{F}\right),
\end{aligned}
$$

where $E_{F}$ is the Fermi energy. Then in atomic units, with $\hbar^{2} / m=1$,

$$
\begin{aligned}
& C_{e 1}=\frac{1}{3} V k_{F} k_{B}^{2} T+\frac{1}{3} A\left[\gamma\left(k_{F}\right)-\frac{1}{4} \pi\right] k_{B}^{2} T, \\
& \chi=\mu_{B}^{2}\left(V / \pi^{2}\right) k_{F}+\mu_{B}^{2}\left(A / \pi^{2}\right)\left[\gamma\left(k_{F}\right)-\frac{1}{4} \pi\right] .
\end{aligned}
$$

The surface contributions are

$$
\begin{aligned}
& C_{\mathrm{e} 1}^{s}=\frac{1}{3} A\left[\gamma\left(k_{F}\right)-\frac{1}{4} \pi\right] k_{B}^{2} T, \\
& \chi^{s}=\left(\mu_{B}^{2} / \pi^{2}\right) A\left[\gamma\left(k_{F}\right)-\frac{1}{4} \pi\right] .
\end{aligned}
$$

The electronic heat capacity, which is always linear in $T$, has the coefficient of $T$ changed by a fraction which is of the same order of magnitude as the ratio of the number of surface atoms to the number of volume atoms. Measurements on samples with high surface areas may allow $C_{\mathrm{eI}}^{s}$ to be determined and thus, within the context of the semi-in- 
finite free-electron model, $\gamma\left(k_{F}\right)$. This phase shift should in turn provide some information about the surface barrier seen by the valence electrons in the metal.
We mention that our results involve only excitations at the Fermi surface, where quasiparticles are well defined, so in this sense our result for $C_{e 1}^{s}$ can be regarded as including many-body effects.
* Present address: Teledyne-Brown, Huntsville, Ala.

${ }^{1}$ R. Denton, B. Mühlschlegel, and D. J. Scalapino, Phys. Rev. Lett. 26, 707 (1.971); Phys. Rev. B $\underline{7}, 3589$ (1973).

${ }^{2}$ N. D. Lang, Solid State Phys. $\underline{28}, 225$ (1.973); see Eq. (A10).

${ }^{3}$ N. D. Lang and W. Kohn, Phys. Rev. B 1, 4555 (1.970); $\underline{3}, 1.215$ (1.971).
${ }^{4}$ When we say that (1) holds for a semi-infinite free-electron model, we really mean that it holds in a slab with a large area $\frac{1}{2} A$ for each surface and a large thickness $V /\left(\frac{1}{2} A\right)$. In a semi-infinite crystal $V$ and $A$ are actually infinite, of course.

${ }^{5} \mathrm{C}$. Kittel, Introduction to Solid State Physics, 4th ed. (Wiley, New York, 1.971), pp. 252 and 51.9. 